\title{
Vasoactive intestinal polypeptide (VIP) corrects chronic inflammatory response syndrome (CIRS) acquired following exposure to water-damaged buildings
}

\author{
Ritchie C. Shoemaker ${ }^{1 *}$, Dennis House ${ }^{1}$, James C. Ryan ${ }^{2}$ \\ ${ }^{1}$ Center for Research on Biotoxin Associated Illnesses, Pocomoke City, USA; *Corresponding Author: ritchieshoemaker@msn.com \\ ${ }^{2}$ Proteogenomics, LLC, Vero Beach, USA
}

Received 17 January 2013; revised 20 February 2013; accepted 27 February 2013

\begin{abstract}
Exposure in water-damaged buildings (WDB) to airborne bioaerosols including metabolic products of toxigenic fungi, bacteria and actinomycetes; and inflammagens, can lead to a persistent innate immune inflammatory illness. This illness, termed a chronic inflammatory response syndrome (CIRS-WDB), is systemic with symptoms acquired from multiple organ systems. Treatment of CIRS-WDB has progressed rapidly as a better understanding of the inflammatory pathophysiology has led to targeted, sequential therapies. The fundamental basis of uncontrolled innate immune responses, the humoral deficiency of regulatory neuropeptides melanocyte stimulating hormone (MSH) or vasoactive intestinal polypeptide (VIP), seen in over $98 \%$ of patients, has not consistently responded to any treatment modality. Use of replacement VIP has been attempted anecdotally; VIP replacement therapies show promise in short term studies but longer therapies have not been attempted. Here we report an open label trial of 20 patients with refractory CIRS-WDB illness who took replacement VIP in a nasal spray for at least 18 months with confirmation of durable efficacy and absence of significant side effects. These 20 patients were similar in symptoms and lab findings to three previously published cohorts involving 1829 patients and 169 controls. Dosage of VIP was titrated downwards from four to zero doses a day to determine minimum effective dose, and re-titrated upwards for maximum improvement over time. The trial showed that VIP therapy safely 1) reduced refractory symptoms to equal controls; 2) corrected inflammatory parameters C4a, TGF beta-1, VEGF, MMP9; 3)
\end{abstract}

corrected estradiol, testosterone and 25-OH Vitamin D; 4) returned pulmonary artery systolic pressure (PASP) during exercise to normal; and 5 ) enhanced quality of life in $100 \%$ of trial patients. Subsequent identification of correction of T-regulatory cell levels supports the potential role of VIP in both innate and adaptive immune function.

Keywords: Vasoactive Intestinal Polypeptide (VIP); Chronic Inflammatory Response Syndrome (CIRS); TGF Beta-1; C4a; MSH; T Regulatory Cells;

Water-Damaged Buildings

\section{INTRODUCTION}

Treatment of the multisystem, multisymptom illness acquired following exposure to the interior environment of buildings with a history of water intrusion and subsequent microbial growth (i.e. WDB) remained problematic until recent years because 1) no consistent pathophysiological mechanisms had been demonstrated; and 2) no biomarkers were consistently identified thereby preventing confirmatory laboratory diagnosis. Various alternative diagnoses have been ascribed to patients with this illness, including medically uncertain illness, fibromyalgia, and "Sick Building Syndrome". Following publication of reports from the US GAO in 2008 [1], WHO in 2009 [2] and the Consensus Statement of Treating Physicians in 2010 [3], the role of chronic inflammation as a response to exposure has been recognized, providing for effective therapies to control most illness parameters. In some cases, however, these therapies did not provide protection from relapse upon reexposure to WDB; some patients remained severely affected.

This illness has been called a chronic systemic inflammatory illness (CIRS-WDB, [3]), paralleling a similar 
description of ciguatera, a biotoxin-associated illness [4] caused by consumption of reef-dwelling, ciguatoxic fish. In three case series of patients ( $\mathrm{N}=1829)$ with CIRSWDB, deficiency of the regulatory neuropeptide vasoactive intestinal polypeptide (VIP) occurred in $98 \%$ of patients $[5,6]$ while less than $10 \%$ of controls demonstrated this deficiency. These data are consistent with nearly 5000 other CIRS-WDB patients and 850 control patients documented in a single clinical practice (RCS).

VIP is a regulatory neuropeptide with diverse physicologic effects. Following activation of outer membranebased receptors, VIP is known to raise cAMP [7]; lower pulmonary artery systolic pressure (PASP) responses to exercise [8]; block peripheral innate immune activation [9]; raise VEGF [10]; restore circadian rhythm [11]; regulate dendritic cells [12]; regulate Th17 function in autoimmunity [13]; and enhance IL-10 production [14]; VIP can also raise peripheral blood levels of CD4+CD25+ T regulatory (Treg) cells [15]; prevent development of an experimental model of rheumatoid arthritis in mice [16] and inhibit TGF beta-1 production by macrophages [17]. Inhaled VIP use has also corrected inflamematory markers seen in patients with sarcoid [18].

In addition to deficiency of VIP, patients with CIRSWDB will invariably have 1) deficiencies of another regulatory neuropeptide, melanocyte stimulating hormone (MSH) in plasma; 2) dysregulated inflammatory responses; and usually have 3) evidence of cellular immune abnormalities marked by elevated TGF beta- 1 in plasma and reduced levels of Tregs in whole blood; 4) abnormal plasma levels of a split product of complement activation, C4a; 5) dysregulation of serum ACTH/cortisol, androgens and ADH/osmolality; 6) abnormal plasma levels of VEGF; 7) disorders of coagulation parameters seen in von Willebrand's profiles; 8) presence of genomically active, multiple antibiotic resistant coagulase negative staphylococci (MARCoNS) in deep aerobic nasal spaces and 9) an abnormal rise in PASP in exercise. Treatment of CIRS-WDB involves multiple sequential steps (Figure 1) that are aimed at 1 ) reducing inflammatory responses and 2) restoring normal hormonal relationships, but these efforts will rarely restore levels of MSH and VIP to normal. Use of VIP in a 28-day study has successfully been employed to restore immunoregulation in sarcoid patients (Prasse et al. 2010), in part by lowering TNF and raising Treg cells. It is the potential benefit from exogenous VIP that demonstrates restoration of innate immune regulation that prompted a pilot study at our clinic beginning in 2008.

\section{METHODS}

Human research approval for this study was provided by Copernicus Group IRB, Cary, NC in 2010. After pro- viding informed consent, 20 patients (11 Caucasian females, mean age 51.1; and 9 Caucasian males, mean age 48.2) with clinically-confirmed CIRS-WDB refractory to all prior treatment modalities (steps $1-10$, as described below, in Figure 1) were enrolled in this trial. All patients were confirmed to be exposed to WDB by observation of illness acquisition solely following water intrusion followed by presence of either 1) visible microbial growth; 2) speciation of molds by QPCR DNA testing; or 3) musty smells. All patients met the case criteria established by the GAO (US GAO 2008). Patients were well known to the clinic with a 36 month mean duration of prior treatment following exposure to WDB. No patients had been treated with VIP previously. Entry criteria included a rise in PASP in exercise as measured by stress echocardiography that exceeded $8 \mathrm{mmHg}$ compared to resting PASP.

For the experimental protocol, subjects self-administered 50 mcg VIP (Aviptadil; Bachem AG, Switzerland) four times a day via nasal aerosol and returned to the clinic to review symptoms and clinical course; and undergo interval physical exam and laboratory testing at scheduled intervals. Clinical data were collected before any treatments, at baseline (BASE); after all other treatments and before VIP (AC2); after 12 months of VIP (12 $\mathrm{M})$; and after 18 months of VIP (18 M). Data collection included physician recorded symptoms in a medical history; levels of VIP, MSH, C4a, TGF beta-1, VEGF, MMP9, estradiol, testosterone, 25-OH vitamin D, lipase, $\mathrm{CBC}$ and CMP. Measurement of CD4+CD25+ T regulatory (Treg) cells was not available at BASE; Treg testing was performed at AC2 and 18 month time periods. Laboratory testing for all analytes was performed by high complexity CLIA-certified labs including LabCorp and Quest Diagnostics.

All data were compared to either established normative values or values from prior testing of healthy control populations $(\mathrm{N}=850)$ performed at this clinic. Data were analyzed by two-sample T-tests for each of two study time points and one-sample T-tests that compared the specified data to the corresponding historical control

\section{Treatment steps}

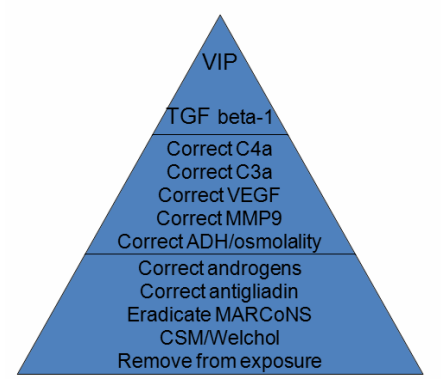

Figure 1. Sequential treatment steps. 
values. A Bonferroni correction to the p-values was done to control the experiment-wise error rate. A p value of $<$ 0.001 was chosen as the determinant of significance for the study.

\section{STRESS ECHOCARDIOGRAPHY}

Standard Bruce protocols were employed during stress echocardiography testing. Target heart rate of $85 \%$ of maximum was obtained after baseline recording showed no evidence of PASP greater than $30 \mathrm{mmHg}$ or left ventricular ejection fraction of less than $50 \%$. All patients achieved target heart rate; none developed ischemic changes on EKG or chest pain during stress testing. Each had a repeat measurement of the tricuspid regurgitation jet (TR) immediately after exercise with recordings of TR performed within 30 seconds of cessation of exercise. Pulmonary artery pressure was calculated from the measurement of the TR based on a standard nomogram.

\section{RESULTS}

Patients tolerated the drug well and there were no dropouts due to adverse effects over the course of the 18 month study. Cases were similar at baseline to known cases of CIRS-WDB in large cohorts previously published $[5,6]$ with excessive numbers of symptoms in cases compared to controls (Table 1) and multiple laboratory abnormalities (Table $\mathbf{1}$ ) in cases compared to controls. This cohort is clearly affected by an innate immune inflammatory process with nearly 23 health symptoms at baseline; marked reduction in VIP and MSH; significant elevation of C4a, MMP9 and TGF beta-1; evidence of increased activity of aromatase with reduction of testosterone and elevated estradiol in males but not females; and reduced Vitamin D3 levels. Lipase levels did not rise in this cohort during the trial.

Use of replacement doses of the regulatory neuropeptide VIP in a nasal spray 1) safely reduced refractory symptoms to control levels; 2) corrected inflammatory parameters to be not significantly different from controls; 3) raised levels of VIP and MSH; 4) returned PASP during exercise to normal; and 5) enhanced quality of life in 20 patients in an open-label trial. Follow-up as long as 18 months after initiation of the study showed durable salutary responses, without significant adverse side effects. Labs and symptoms showed continued improvement over time to become similar to controls (Table 2 and Figure 2) although MSH and VIP continued to be significantly depressed compared to controls. Elevated PASP in stress exercise testing was reduced to $<8 \mathrm{mmHg}$ in all subjects within two months (data not shown). Symptom reduction to equal those of controls occurred in all treated patients. Laboratory results showed a marked improvement over both baseline values and fol- lowing all previously employed therapies (See Table 2, Figure 2).

We have no other studies to use for comparison of results of VIP use as these patients were labeled as refractory to all modalities employed. There are no similar studies of long tem use of VIP in the world's literature.

While significant differences $(\mathrm{p}<0.001)$ were identified between controls and baseline lab values in patients for symptoms, MSH, VIP, MMP9, C4a, TGFb-1, 25-OH Vitamin D and testosterone in males, by the end of the trial patient values were not statistically different from controls, except for VIP and MSH. Flow cytometry showed a significant increase of CD4+CD25+ Treg cells from a mean of 8.9 to 22.5. No changes in CBC and CMP were noted. One patient had a transient elevated level of lipase without abdominal pain that resolved without cessation of use of VIP. After six months patients titrated use of VIP to these symptoms with verification of benefit obtained by clinical exam and blood testing at three month intervals. Eight patients consistently used VIP at three or four times a day; four patients used VIP once or twice a day. Three patients used VIP before strenuous activity only; five patients stopped using VIP at various points during the trial as they had correction of symptoms and no evidence of relapse without protocol medications. For these patients, only data obtained while using VIP was subjected to statistical calculation.

\section{DISCUSSION}

VIP is a 28 amino acid regulatory neuropeptide that has multiple salutary health effects. This study was designed to assess the benefits and potential risks of VIP use in severely affected patients who had previously completed all sequential treatments while attempting to correct the above illness parameters but were still quite ill (Table 2). Each patient was treated sequentially in 30-day steps by 1) removal from exposure; 2) treatment with cholestyramine for at least one month and ongoing;

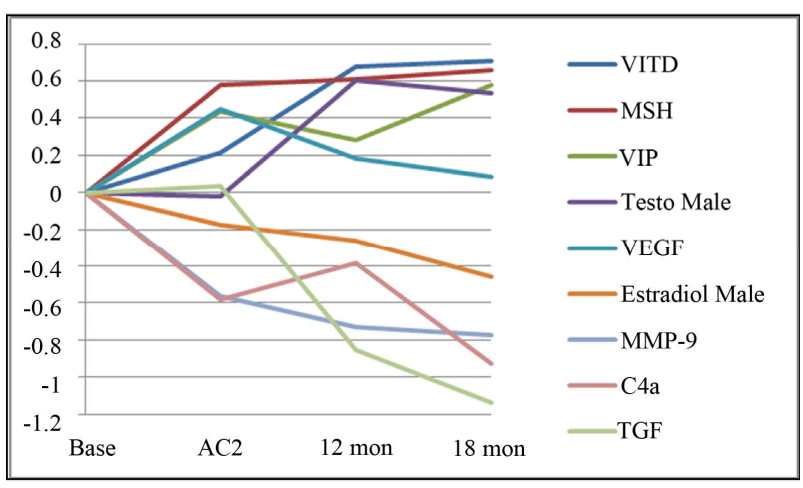

Figure 2. Mean values of labs with significant differences between baseline and controls. Values from Table 1 were log transformed and normalized to baseline. 
Table 1. Prior studies on cohorts of CIRS-WDB patients.

\begin{tabular}{|c|c|c|c|c|c|c|c|c|c|c|c|c|c|}
\hline & $\begin{array}{c}2007 \\
\text { Control }\end{array}$ & $\begin{array}{c}2007 \\
\text { Control }\end{array}$ & $\begin{array}{c}\text { 2009/2010 } \\
\text { Control }\end{array}$ & $\begin{array}{c}\text { 2009/2010 } \\
\text { Control }\end{array}$ & $\begin{array}{l}2007 \\
\text { Case }\end{array}$ & $\begin{array}{l}2007 \\
\text { Case }\end{array}$ & 2007 & $\begin{array}{l}2009 \\
\text { Case }\end{array}$ & $\begin{array}{l}2009 \\
\text { Case }\end{array}$ & 2009 & $\begin{array}{l}2010 \\
\text { Case }\end{array}$ & $\begin{array}{l}2010 \\
\text { Case }\end{array}$ & 2010 \\
\hline & $\mathrm{N}$ & Mean & $\mathrm{N}$ & Mean & $\mathrm{N}$ & Mean & p value & $\mathrm{N}$ & Mean & p value & $\mathrm{N}$ & Mean & $\mathrm{p}$ value \\
\hline Age & 37 & 51.1 & 132 & 50.3 & 202 & 46.1 & 0.027 & 812 & 47.8 & 0.0378 & 815 & 49.2 & \\
\hline Symptoms & 37 & 2.5 & 132 & 2.9 & 2.2 & 22.9 & $<0.0001$ & 812 & 22.7 & $<0.0001$ & 815 & 22.6 & $<0.001$ \\
\hline VIP & 37 & 41.1 & 132 & 36.6 & 199 & 7.8 & $<0.0001$ & 812 & 12.8 & $<0.0001$ & 815 & 12.8 & $<0.001$ \\
\hline MSH & 37 & 34.7 & 132 & 41.2 & 201 & 14.1 & $<0.0001$ & 799 & 13.1 & $<0.0001$ & 815 & 13.1 & $<0.001$ \\
\hline Leptin & 36 & 14.5 & 132 & 17.9 & 197 & 27.9 & 0.546 & 697 & 19.3 & 0.816 & 815 & 19.4 & 0.816 \\
\hline $\mathrm{ADH}$ & 34 & 3.9 & 132 & 3.7 & 192 & 3.3 & 0.709 & 692 & 3.1 & 0.544 & 815 & 3.1 & 0.544 \\
\hline Osmo & 34 & 288 & 132 & 288 & 200 & 298 & $<0.0001$ & 709 & 302 & $<0.0001$ & 815 & 302 & $<0.001$ \\
\hline АCTH & 35 & 23.2 & 132 & 22 & 200 & 15.8 & 0.084 & 687 & 16.8 & 0.0007 & 815 & 15.8 & $<0.001$ \\
\hline Cortisol & 36 & 16.8 & 132 & 21.7 & 201 & 15.4 & 0.811 & 689 & 13 & 0.0009 & 815 & 12.9 & $>0.5$ \\
\hline MMP-9 & 37 & 284 & 132 & 269 & 201 & 376 & 0.04 & 812 & 420 & $<0.0001$ & 815 & 419 & $<0.001$ \\
\hline PAI-1 & 37 & 6.63 & 132 & 6.3 & 182 & 5.72 & 0.561 & 636 & 7 & 0.6605 & 815 & 21 & $<0.001$ \\
\hline VEGF & 30 & 67.2 & 132 & 67.9 & 198 & 64.7 & 0.888 & 656 & 59 & 0.4084 & 815 & 62.2 & 0.41 \\
\hline C3A & 24 & 242 & 132 & 253 & 185 & 929 & 0.24 & 808 & 435 & $<0.0001$ & 815 & 427 & $<0.001$ \\
\hline C4A & 22 & 1852 & 132 & 2303 & 190 & 8818 & $<0.001$ & 812 & 9661 & $<0.0001$ & 815 & 9924 & $<0.001$ \\
\hline TGF Beta-1 & & & 132 & 2365 & & & & 812 & 6412 & $<0.0001$ & 815 & 4602 & $<0.001$ \\
\hline CRP & 35 & 2.6 & 132 & 2.4 & 187 & 5.12 & 0.246 & 812 & 1.8 & 0.424 & 815 & 1.9 & 0.424 \\
\hline ESR & 37 & 8 & 132 & 9 & 187 & 27.9 & $<0.0001$ & 792 & 6 & $>0.5$ & 815 & 4 & $>0.5$ \\
\hline LgE & 36 & 49 & 132 & 46 & 191 & 82.5 & 0.732 & 802 & 39 & $>0.5$ & 815 & 41 & $>0.5$ \\
\hline $\mathrm{CBC}$ & 41 & 0 & 132 & 5 & 202 & 7 & 0.999 & 812 & 5 & 0.999 & 815 & 5 & 0.999 \\
\hline СMP & 41 & 0 & 132 & 5 & 202 & 15 & 0.999 & 812 & 6 & 0.999 & 815 & 5 & 0.999 \\
\hline Vwf & 41 & & 132 & 11 & 191 & 16 & $<0.001$ & 704 & 67 & $<0.001$ & 815 & 71 & $<0.001$ \\
\hline ACLA & 41 & 4 & 132 & 13 & 199 & 36 & $<0.001$ & 812 & 24 & $<0.001$ & 815 & 23 & $<0.001$ \\
\hline AGA & 41 & 5 & 132 & 9 & 200 & 16 & $<0.001$ & 812 & 32 & $<0.001$ & 815 & 17 & $<0.001$ \\
\hline
\end{tabular}

Table 2. Symptoms and laboratory mean values \pm standard deviation for patients. Sx = symptoms, Estra-F = estradiol in females, Estra-M = estradiol in males, Test = testosterone. Shaded cells indicate significance between control values.

\begin{tabular}{cccccc}
\hline LAB & Control & Base & AC2 & $\mathbf{1 2} \mathbf{~ m o n}$ & $\mathbf{1 8} \mathbf{~ m o n}$ \\
\hline SX & 2.9 & 29.8 & 17.8 & 5.5 & 3.4 \\
VIP pg/ml & 28.9 & 9.3 & 14.4 & 12.3 & 16.6 \\
MSH pg/ml & 37.2 & 9.4 & 16.8 & 17.3 & 18.2 \\
C4a ng/ml & 2830 & 8346 & 4662 & 5679 & 3306 \\
TGF ng/ml & 2380 & 12,579 & 13,015 & 5359 & 4040 \\
VEGF pg/ml & 65 & 61 & 95.3 & 73.4 & 66.2 \\
MMP-9 ng/ml & 332 & 628 & 358 & 302 & 290 \\
Estra-F mg/ml & 29.8 & 28 & 26.6 & 27.8 & 28.9 \\
Estra-M mg/ml & 23.1 & 36 & 30.3 & 27.7 & 22.8 \\
Test-F mg/ml & 28.2 & 20 & 18.9 & 22.6 & 25.2 \\
Test-M & 415 & 298.8 & 293.5 & 546.7 & 511.5 \\
VIT D Int Units & 38.4 & 19.4 & 24.1 & 38.3 & 39.3 \\
Lipase mg/ml & 30.1 & 24.2 & 30.8 & 30.7 & 27.9 \\
\hline
\end{tabular}

3) eradication biofilm-forming coagulase negative staphylococci; 4) discontinuance of consumption of gluten if they had a positive antigliadin antibody titer (three months minimum required); 5) correction of abnormalities androgens; 6) correction of abnormalities in regulation of salt and water with synthetic desmopressin as shown by simultaneous ADH and osmolality; 7) normalizing MMP9 with pioglitazone 45 mg daily; 8) normalizing VEGF with high dose omega-3 fish oils (4.2 grams daily); 9) correction of C3a with high dose statins; 10) attempting to correct C4a with erythropoietin but only if entry criteria met; 11) attempting to correct TGF beta-1 with losartan $25 \mathrm{mg}$ daily, monitoring blood pressure carefully. Our initial use of low dose VIP by nasal instilllation was shown to be safe in human volunteers; its use provided prompt reduction in symptoms and blunted accentuated pulmonary artery responses to exercise. However, benefit of replacement VIP in earlier trials was not observed to be universal. Presence of any one of three parameters was associated with reduced efficacy: 1) depressed visual contrast sensitivity (VCS); 2) measure- 
ment of fungal DNA in settled dust using QPCR that resulted in an Environmental Relative Mold Index (ERMI) $>2$; and 3) presence of multiply antibiotic resistant biofilm-forming coagulase negative staphylococci (MARCoNS) in deep nasal aerobic spaces. Patients with these findings were excluded from the formal replacement trial.

Current therapies for CIRS-WDB can improve quality of life in patients, but symptom reduction and correction of inflammatory responses alone do not necessarily lead to full restoration of normal immune responses. The restoration of appropriate neuropeptide levels is critical for proper regulation of hormonal and immune system inflammatory responses. VIP replacement demonstrated its value in the correction of such regulation during this trial. Even though levels of MSH and VIP did not return to control values, they doubled compared to baseline, with additional improvement after all prior therapies were completed.

An important anti-inflammatory mechanism of VIP is its ability to generate tolerogenic dendritic cells, which in turn induce additional $\mathrm{T}$ regulatory cells that can suppress Th1 responses, generating a Th2 response and transfer such suppression to naïve hosts [19]. Plasmacytoid dendritic cells have receptors for VIP; when incubated in vitro with VIP these cells showed inhibition of production of interferon alpha and interferon gamma/IL-4, reflecting skewing of these cells to a Th2 expression. VIP therefore may be involved with down-regulating Th1 responses [19].

This study confirms our hypotheses that use of VIP in VIP-deficient patients is both durably safe and effective for up to 18 months. When used by nasal instillation VIP is well-tolerated, has few side effects and is unlikely to result in an over-dosage. Treatment with VIP restored clinical functioning in a cohort of CIRS-WDB patients with severe illness characterized by profound, refractory abnormalities in innate immune inflammatory responses (see BASE on Table 2). Symptom improvement was remarkable, including correction of chronic fatigue, shortness of breath and asthma-like conditions, executive cognitive deficits, neurologic symptoms and chronic joint pain. We confirm here the known effects of VIP in the normalization of Treg levels and controlling cytokine responses. Use of VIP corrected abnormal PASP responses during exercise; reduced total number of symptoms to equal controls; and down-regulated inflammatory responses as measured by MMP9, TGF beta- 1 and C4a levels, with positive effects occurring over time. The benefit of restoration of androgen levels to equal control levels suggests a down-regulating effect of VIP on the enzyme aromatase. The normalization of Vitamin D3 levels was unexpected, suggesting additional but as yet undefined upstream aspects of VIP as a pluripotent im- munoregulatory hormone.

\section{CONCLUSION}

Evaluation of clinical use of VIP will require additional study. A double blinded, placebo controlled clinical trial has begun. The salutary but preliminary results reported here show safety and benefit in severely affected CIRS-WDB patients in an open-label trial. We have now used VIP by nasal instillation in over 600 hundred patients whose clinical findings are similar to those reported here. Now that patients with CIRS-WDB can be identified proteomically, with treatment protocols readily available (Figure 1), further studies are planned to identify possible genomic effects of sequential treatment protocols for WDB-CIRS patients. Specific attention to the genomic changes seen following VIP therapy may add to our understanding of the complexity of the CIRSWDB as proteomic evidence suggests correction of deregulated inflammatory pathways. Application of these proteomic findings to other illnesses characterized by CIRS may be possible.

\section{ACKNOWLEDGEMENTS}

Special thanks to William Meyer III, PhD for comments on the manuscript; and Debbie Waidner and Debra Bell for data processing and collation.

\section{REFERENCES}

[1] US-GAO (2008) GAO-08-980. United States Government Accountability Office: Indoor Mold: Better Coordination of Research on Health Effects and More Consistent Guidance Would Improve Federal Efforts, GAO, Washington DC.

[2] WHO (2009) World Health Organization guidelines for indoor air quality: dampness and mould.

[3] Shoemaker, R., Mark, L. and McMahon, S. (2010) Research Committee report on diagnosis and treatment of chronic inflammatory response syndrome caused by exposure to the interior environment of water-damaged buildings: Expert treating physicians consensus. Policyholders of America, South Carolina, USA.

[4] Shoemaker, R., House, D. and Ryan, J. (2010) Defining the neurotoxin derived illness chronic ciguatera using markers of chronic systemic inflammatory disturbances: A case/control study. Neurotoxicology and Teratology, 32, 633-639. doi:10.1016/j.ntt.2010.05.007

[5] Shoemaker, R. and Maizel, M. (2009) Innate immunity, MR spectroscopy, HLA DR, TGF beta-1, C4a, VIP and capillary hypoperfusion define acute and chronic illness acquired after exposure to water-damaged buildings. Healthy Buildings IX, Syracuse.

[6] Shoemaker, R. and Maizel, M. (2010) Exposure to waterdamaged buildings causes a readily identifiable chronic inflammatory response syndrome that is successfully 
treated by a sequential intervention protocol. Paper Presented at the International Mycological Congress 9, Edinburgh.

[7] Bourgault, S., Vaudry, D., Dejda, A., Doan, N.D., Vaudry, H. and Fournier, A. (2009) Pituitary adenylate cyclaseactivating polypeptide: Focus on structure-activity relationships of a neuroprotective peptide. Current Medicinal Chemistry, 16, 4462-4480. doi:10.2174/092986709789712899

[8] Gupta, V. and Ahsan, F. (2010) Inhalational therapy for pulmonary arterial hypertension: current status and future prospects. Critical Reviews in Therapeutic Drug Carrier Systems, 27, 313-370.

doi:10.1615/CritRevTherDrugCarrierSyst.v27.i4.20

[9] Delgado, M., Gomariz, R.P., Martinez, C., Abad, C. and Leceta, J. (2000) Anti-inflammatory properties of the type 1 and type 2 vasoactive intestinal peptide receptors: Role in lethal endotoxic shock. European Journal of Immunology, 30, 3236-3246. doi:10.1002/1521-4141(200011)30:11<3236::AID-IMM U3236>3.0.CO;2-L

[10] Yu, X.J., Ren, X.H., Xu, Y.H., Chen, L.M., Zhou, C.L. and Li, C.Y. (2010) Vasoactive intestinal peptide induces vascular endothelial growth factor production in human HaCaT keratinocytes via MAPK pathway. Neuropeptides, 44, 407-411. doi:10.1016/j.npep.2010.05.002

[11] Francl, J.M., Kaur, G. and Glass, J.D. (2010) Regulation of vasoactive intestinal polypeptide release in the suprachiasmatic nucleus circadian clock. Neuroreport, 21, 10551059. doi:10.1097/WNR.0b013e32833fcba4

[12] Chorny, A., Gonzalez-Rey, E. and Delgado, M. (2006) Regulation of dendritic cell differentiation by vasoactive intestinal peptide: therapeutic applications on autoimmunity and transplantation. Annals of the New York Academy of Sciences, 1088, 187-194. doi:10.1196/annals.1366.004

\section{APPENDIX}

Acronyms used:

ACLA anticardiolipin antibodies

ACTH adrenocorticotrophic hormone

ADH antidiuretic hormone

cAMP cyclic AMP

CBC complete blood count

CIRS chronic inflammatory response syndrome

C3a split activation product of C3

C4a split activation product of $\mathrm{C} 4$

CMP comprehensive metabolic profile

CSM cholestyramine

ERMI Environmental Relative Moldiness Index

HLA DR human leukocyte antigen Class II, DR locus
[13] Gonzalez-Rey, E. and Delgado, M. (2005) Role of vasoactive intestinal peptide in inflammation and autoimmunity. Current Opinion in Investigational Drugs, 6, 11161123.

[14] Delgado, M., Munoz-Elias, E.J., Gomariz, R.P. and Ganea, D. (1999) Vasoactive intestinal peptide and pituitary adenylate cyclase-activating polypeptide enhance IL10 production by murine macrophages: In vitro and in vivo studies. Journal of Immunology, 162, 1707-1716.

[15] Delgado, M., Chorny, A., Gonzalez-Rey, E. and Ganea, D. (2005) Vasoactive intestinal peptide generates CD4+CD25+ regulatory T cells in vivo. Journal of Leukocyte Biology, 78, 1327-1338. doi:10.1189/jlb.0605299

[16] Delgado, M., Abad, C., Martinez, C., Leceta, J. and Gomariz, R.P. (2001) Vasoactive intestinal peptide prevents experimental arthritis by downregulating both autoimmune and inflammatory components of the disease. $\mathrm{Na}$ ture Medicine, 7, 563-568. doi:10.1038/87887

[17] Leceta, J., Gomariz, R.P., Martinez, C., Carrion, M., Arranz, A. and Juarranz, Y. (2007) Vasoactive intestinal peptide regulates Th17 function in autoimmune inflammation. Neuroimmunomodulation, 14, 134-138. doi:10.1159/000110636

[18] Prasse, A., Zissel, G., Lutzen N., Schupp, J., Schmiedlin, R., Gonzalez-Rey, E., Rensing-Ehl, A., Bacher, G, Cavalli, V., Bevec, D., Delgado, M. and Muller-Quernheim, J. (2010) Inhaled vasoactive intestinal peptide exerts immunoregulatory effects in sarcoidosis. American Journal of Respiratory and Critical, 182, 540-548. doi:10.1164/rccm.200909-14510C

[19] Fabricius, D., O’Dorisio, M.S., Blackwell, S. and Jahrsdorfer, B. (2006) Human plasmacytoid dendritic cell function: Inhibition of IFN-alpha secretion and modulation of immune phenotype by vasoactive intestinal peptide. Journal of Immunology, 177, 5920-5927.

MARCoNS multiply antibiotic resistant coagulase negative staphylococci

MSH alpha melanocyte stimulating hormone

MMP-9 matrix metalloproteinase 9

PASP pulmonary artery systolic pressure

TGF beta- 1 transforming growth factor beta- 1

TNF tumor necrosis factor

$\mathrm{T}$ reg $\mathrm{T}$ regulatory lymphocyte

VCS visual contrast sensitivity

VEGF vascular endothelial growth factor

VIP vasoactive intestinal polypeptide

vWF von Willebrand's profile

WDB water-damaged building 